\title{
Precision Measurements of Parity-Violation in Deep Inelastic Scattering using SoLID
}

\author{
Rakitha S. Beminiwattha ${ }^{* \dagger}$ \\ Department of Physics, Syracuse University \\ rakithabejlab.org
}

\begin{abstract}
A new proposal to measure parity violating deep inelastic asymmetry (PVDIS) will provide a precision test of the Standard Model through an unique constraints on axial-vector neutral electronquark effective couplings and a precision measurement of the weak mixing angle. We plan to measure the asymmetry to about $0.5 \%$ precision over the Bjoken $\mathrm{x}$ from 0.3 to 0.7 where the PV asymmetry is approximately independent of hadronic structure. If any deviations observed, the residual hadronic and new physics contributions in the asymmetry could be separated by kinematic dependence of the PVDIS asymmetry. At the proposed precision level, asymmetry will be sensitive to novel hadronic structure effects including charge symmetry violation (CSV) effects and higher twist effects. Observation of these effects itself will be beneficial to building better theoretical models. A deuterium target will be used for Standard Model tests while a d/u quark distribution ratio measurement is proposed using a hydrogen target.

The broad reach in kinematic and high statistical precision at which PVDIS asymmetries are planned to measure, a large angle acceptance, high luminosity, and large azimuthal acceptance device is a necessity. We are proposing a new spectrometer based on a solenoid magnet called Solenoidal Large Intensity Device (SoLID) that will provide broad range of electroweak and QCD physics measurements to be conducted at the Jefferson Laboratory. The apparatus will have set of tracking gas electron multiplier (GEM) detectors, a gas Cerenkov detector, and a sampling electromagnetic calorimeter to provide particle identification. The experiment will be conducted as a high rate counting mode experiment.
\end{abstract}

The XXIII International Workshop on Deep Inelastic Scattering and Related Subjects

April 27 - May 1, 2015

Southern Methodist University

Dallas, Texas 75275

\footnotetext{
*Speaker.

${ }^{\dagger}$ SoLID Collaboration
} 


\section{Overview}

Charge neutral electron-quark scattering can take place by exchanging a $Z^{0}$ or a $\gamma$ boson. The electron-quark neutral current scattering cross section dependents on the helicity of the electron due to the parity violating (PV) weak $\mathrm{Z}^{\circ}$ boson exchange (see Figures 1 and 2). Therefore electron-quark effective couplings can be extracted by measuring the differential helicity correlated scattering cross section (PV asymmetry) as shown in Eq. 1.1.

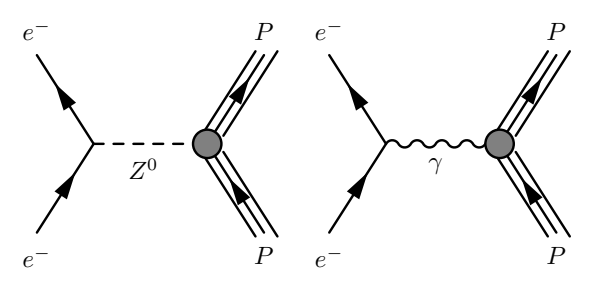

Figure 1: Electron-quark scattering can take place by exchanging a $\mathrm{Z}^{\mathrm{o}}$ or a $\gamma$ boson.

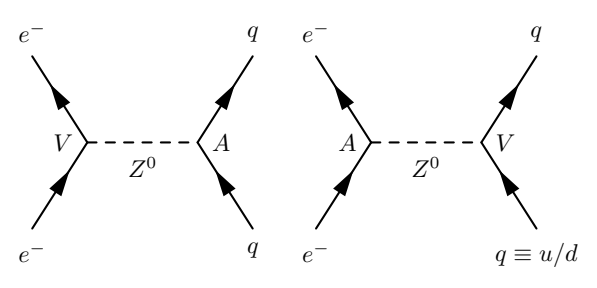

Figure 2: $Z^{0}$ boson exchange either via axial-vector (A-V) quark-electron or vector-axial (V-A) quark-electron interactions.

$$
A_{L R}=\frac{\frac{d \sigma^{R}}{d \Omega}-\frac{d \sigma^{L}}{d \Omega}}{\frac{d \sigma^{\mathrm{R}}}{d \Omega}+\frac{d \sigma^{L}}{d \Omega}} \propto \frac{M^{E M} \cdot M^{N C}}{\left|M^{E M}\right|^{2}}
$$

where the $\frac{\mathrm{d} \sigma^{\mathrm{R} / \mathrm{L}}}{\mathrm{d} \Omega}$ is the helicity (Left/Right) dependent cross sections, $\mathrm{M}^{\mathrm{EM}}$ is the electromagnetic scattering amplitude, and $\mathrm{M}^{\mathrm{NC}}$ is $\mathrm{PV}$ weak neutral scattering amplitude.

The parity violating deep inelastic (PVDIS) asymmetry in the kinematic range of $\mathrm{Q}^{2}>>$ $1 \mathrm{GeV}^{2}$ and $\mathrm{W}^{2}>4 \mathrm{GeV}^{2}$ can be derived in terms of effective electron-quark couplings and other kinematics factors.

$$
\mathrm{A}_{\mathrm{PV}}^{\mathrm{DIS}}=\frac{\mathrm{G}_{\mathrm{F}} \mathrm{Q}^{2}}{4 \sqrt{2} \pi \alpha}\left[\mathrm{a}_{1}\left(\mathrm{x}_{\mathrm{bjk}}\right) \mathrm{Y}_{1}+\mathrm{a}_{3}\left(\mathrm{x}_{\mathrm{bjk}}\right) \mathrm{Y}_{3}(\mathrm{y})\right]
$$

where $\mathrm{Y}_{1}(\mathrm{y}) \simeq 1$ and $\mathrm{Y}_{3} \simeq \frac{1-(1-\mathrm{y})^{2}}{1+(1-\mathrm{y})^{2}}$ and $\mathrm{y}=\frac{v}{\mathrm{E}}, v=\mathrm{E}-\mathrm{E}^{\prime}$ where $\mathrm{E}$ and $\mathrm{E}^{\prime}$ are incident and scattered electron energies, respectively. The term $a_{1}\left(x_{b j k}\right)$ includes axial-vector (V-A) electronquark coupling constants while term $a_{3}\left(x_{b j k}\right)$ includes vector-axial (A-V) electron-quark coupling constants. $\mathrm{x}_{\mathrm{bjk}}$ is the Bjorken scaling of the parton distribution functions (PDFs).

For an isoscalar (deuterium) target, PVDIS asymmetry is independent of PDFs, $\mathrm{x}_{\mathrm{bjk}}$ and W. The standard model (SM) predicts $\mathrm{Q}^{2}$ and y variations for the PVDIS asymmetry and for a deuterium target $\mathrm{a}_{1}\left(\mathrm{x}_{\mathrm{bjk}}\right)$ and $\mathrm{a}_{3}\left(\mathrm{x}_{\mathrm{bjk}}\right)$ are given in eq 1.3 .

$$
\begin{aligned}
& \mathrm{a}_{1}^{\mathrm{D}}\left(\mathrm{x}_{\mathrm{bjk}}\right)=\frac{6}{5}\left(2 \mathrm{C}_{1 \mathrm{u}}-\mathrm{C}_{1 \mathrm{~d}}\right)\left(1+\frac{0.6 \mathrm{~s}^{+}}{\mathrm{u}^{+}+\mathrm{d}^{+}}\right) \\
& \mathrm{a}_{3}^{\mathrm{D}}\left(\mathrm{x}_{\mathrm{bjk}}\right)=\frac{6}{5}\left(2 \mathrm{C}_{2 \mathrm{u}}-\mathrm{C}_{2 \mathrm{~d}}\right)\left(\frac{\mathrm{u}^{-}+\mathrm{d}^{-}}{\mathrm{u}^{+}+\mathrm{d}^{+}}\right)+\cdots
\end{aligned}
$$

Where $f_{i}^{ \pm}=f_{i} \pm \bar{f}_{i}$ are quark anti-quark flavor combinations. 
The higher order quantum chromodynamics (QCD) effects can still manifest and perturb the PVDIS asymmetry. These effects originate from flavor dependent quark distributions ( $\mathrm{u}, \mathrm{d}$, and $\mathrm{s}$ ), charge symmetry violations (CSV), and nuclear medium effects which will be further discussed in the next section.

\section{Physics Motivations}

PVDIS asymmetries provide attractive features to perform precise SM tests at broad kinematic ranges provided they are measured at large $\mathrm{Q}^{2}$ values to gain the advantage of large PV asymmetry amplitude. The physics motivations for PVDIS asymmetry measurements include but not limited to a precision test of the standard model, a search for CSV effects, a test of QCD higher twist corrections and a test of nuclear medium effects on quark flavors.

The running of the weak mixing angle is defined as the SM prediction for the value of the weak mixing angle over broad energy scale based on precise determination of it at $\mathrm{z}$ boson resonance. A set of low energy measurements of the weak mixing angle has verified the running in low energy scales (see Figure B). The precision reachable by the SoLID-PVDIS will provide better understanding of the NuTeV anomaly that is predicted to be coming from CSV effects in high Z nuclear medium. It will also provide a test of potential low energy dark sector parity violating interactions that appear as a shift in weak mixing angle measurement from the SM prediction [1].

The present constraints on axial-vector and vector-axial electron-quark effective coupling constants are based on most recent parity violating electron scattering (PVES) and atomic parity violaitng (APV) measurements (see Figure $\emptyset$ left panel). These results are in agreement with the SM predictions. The experimental constraints on the effective coupling constants can be used to deduce mass reach of new PV physics interactions (see Figure $\Theta$ right panel). A combined search based on a precision SoLID-PVDIS measurement and final results from the Qweak [2] experiment can increase the sensitivity to PV new physics interactions at higher mass scales.

SoLID PVDIS experiment will measure the asymmetry measurements in broad kinematic range that will allow to separate new physics and hadronic physics. Hadronic interactions at play in PVDIS asymmetry depend critically on Bjorken scaling parameter $\left(\mathrm{x}_{\mathrm{bjk}}\right)$ and $\mathrm{Q}^{2}$ while potential new physics interactions will depend only with kinematic parameter $\mathrm{y}=\frac{v}{\mathrm{E}}$. The PVDIS asymmetry measurements will provide the first direct observation of parton level charge symmetry violation (CSV) effects on a deuterium target. When additional nuclear medium modifications such as EMC effects in heavy nuclei (neutron rich) are considered, there will be modifications to the parton distribution functions (PDFs) in heavier nuclei [7]. PVDIS asymmetries on heavy neutron rich targets provide a precision tool to access nuclear medium modifications to PDFs. There is an on-going effort to produce a physics proposal to SoLID experiment to measure PVDIS asymmetries on high $\mathrm{Z}$ nuclei (such as ${ }^{48} \mathrm{Ca}$ ). By measuring PVDIS asymmetry on a proton target a set of $d / u$ ratio measurement as a function of $x_{\mathrm{bjk}}$ can be extracted independent of any nuclear medium corrections which will provide complementary data to proposed ${ }^{3} \mathrm{H}$ and ${ }^{3} \mathrm{He}$ form factor ratio measurements proposed at JLab [8, 9]. When transitioning from simple quark parton model (QPM) to QCD based PVDIS asymmetries, the higher order terms in operator product expansion (OPE) must be included that will introduce higher twist (HT) effects to the PVDIS asymmetries [10]. In deuterium PVDIS asymmetry, only HT effects due to quark-quark correlations are contributing while all other con- 


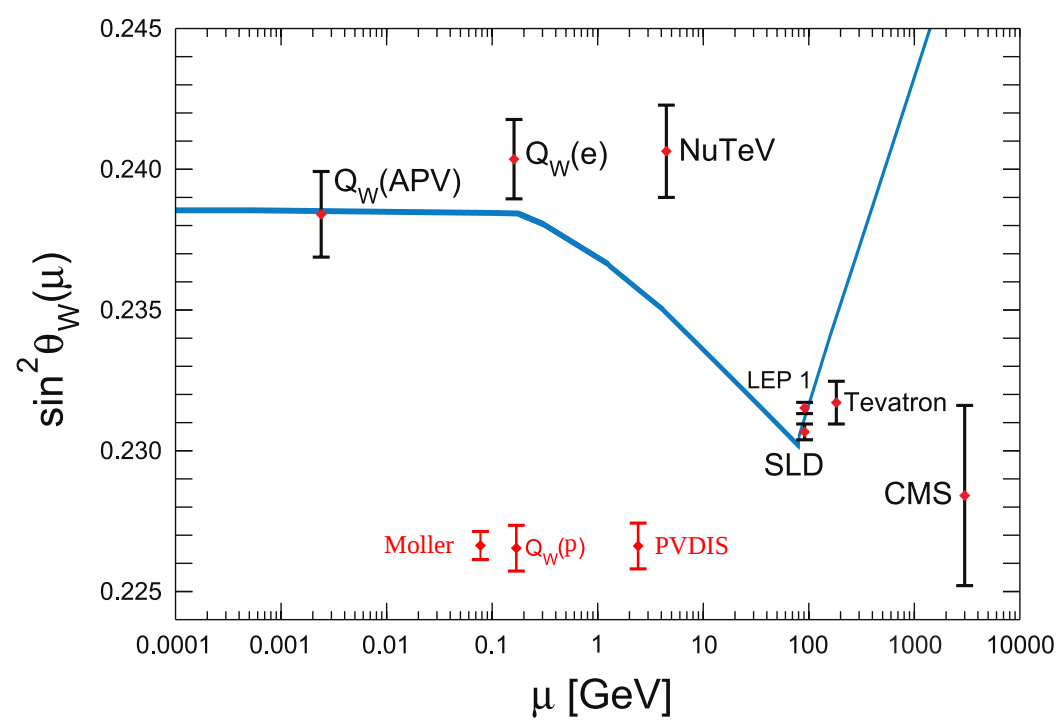

Figure 3: The "running of $\sin ^{2} \theta_{\mathrm{W}}$ " in the $\overline{\mathrm{MS}}$ scheme is compared with low energy measurements of the $\sin ^{2} \hat{\theta}_{\mathrm{W}}(\mu)$ (from [3]). The Qweak [2] ( $\mathrm{Q}_{\mathrm{w}}(\mathrm{p})$ ) from Jlab and the $\mathrm{Q}_{\mathrm{W}}(\mathrm{e})$ from SLAC E158 [⿰亻也 m measurements are done at the same $\mu$-scale. The original NuTeV result [5] is shown but once finalized, new corrections are expected to reduce the $\mathrm{NuTeV}$ deviation from the SM [3, pages 140-141]. Proposed measurements from SoLID-PVDIS, $12 \mathrm{GeV}$ Møller experiment [6], and expected final results from Qweak are shown in red color.

tributors cancel out. Therefore analyzing PVDIS asymmetry measurements in a broad kinematic range, the quark-quark HT effects can be isolated from quark-gluon HT effects. In summary, PVDIS measurements will use $0.4<\mathrm{x}_{\mathrm{bjk}}$ and high $\mathrm{Q}^{2}$ data to test the SM. It will cover broad $\mathrm{Q}^{2}$ range for $0.3<\mathrm{x}_{\mathrm{bjk}}<0.6$ to contraint $\mathrm{HT}$ effects and will search for CSV effects by using the $\mathrm{x}_{\mathrm{bjk}}$ dependence of PVDIS asymmetry.

\section{SoLID Apparatus}

PVDIS asymmetry measurements will require high intensity polarized electron beam which is avilable at Jefferson lab but lacks a large acceptance broad kinematic apparatus requried by the PVDIS physics program. A new apparatus is proposed called Solenoidal Large Intensity Device (SoLID) where a target is kept at the center of a solenoidal magnet and set of tracking detectors, a gas Cerenkov detector and an electromagnetic calorimeter provide azimuthally symmetric particle detection at very high rates [12].

A set of lead baffles inside the solenoid coupled with the solenoid magnetic field provide collimation and background reduction [12]. The conceptual design of the SoLID apparatus is shown in Figure 5. To reach the required figure-of-merit (see Figure 6) on PVDIS measurements, it will need about $85 \%$ polarized $50 \mu \mathrm{A}$ at $11 \mathrm{GeV}$ electron beam on the target (about $1 \times 10^{39} \mathrm{~cm}^{-2} \mathrm{~s}^{-1}$ luminosity). The large scattering angle range from $22^{\circ}$ to $35^{\circ}$ will provide scattered electrons in the range of $0.25<\mathrm{x}_{\mathrm{bjk}}<0.75$. PVDIS program will use moderate running times of about 3 months at $11 \mathrm{GeV}$ to simultaneously measure PVDIS asymmetries to about $1 \%$ precision over this broad 

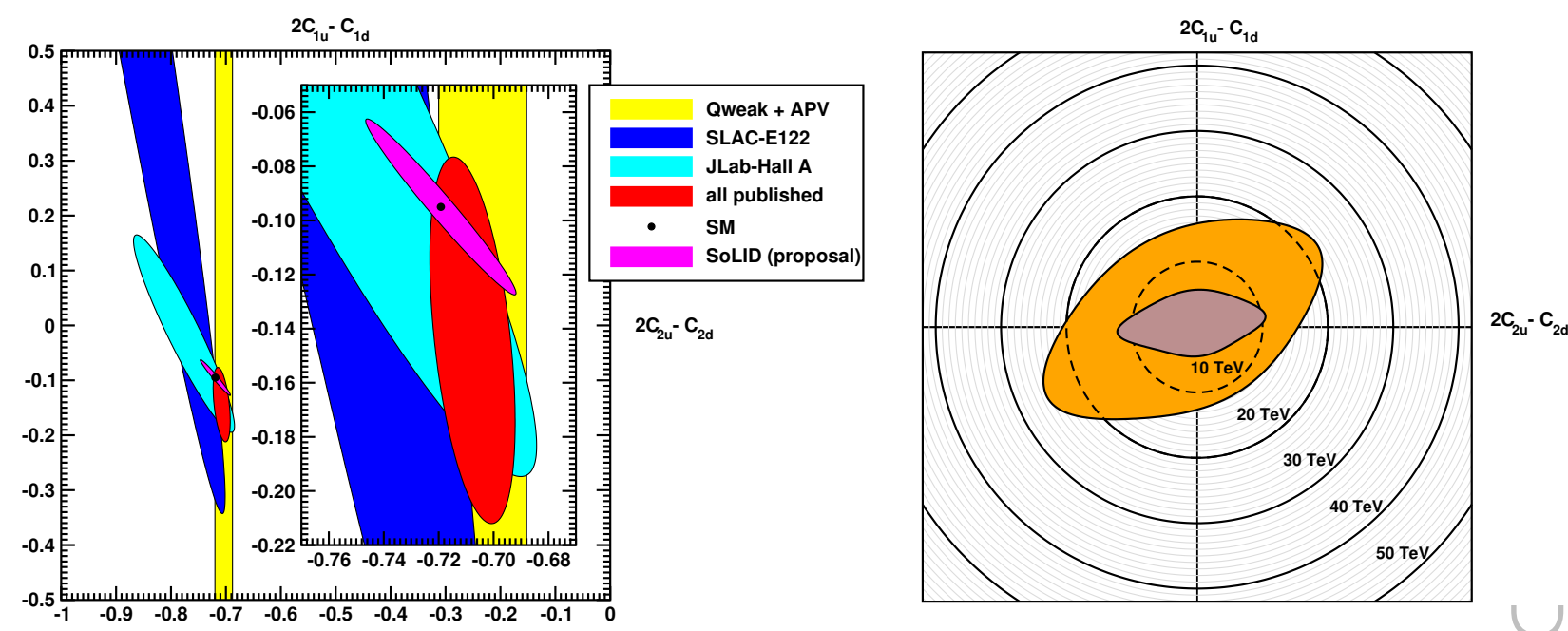

Figure 4: Axes are linear combinations of axial-vector quark-electron and vector-axial quark-electron effective coupling constants. Left: The phase-space of the axial-vector and vector-axial electron-quark effective coupling constants can be constraints by using SoLID PVDIS measurements combined with other precision measurements. Present measurements predict non zero electron-quark coupling constants and an agreement with the SM predictions [11]. Right: A polar plot of the mass scales of new parity violating physics interactions assuming a new physics coupling strength of $\mathrm{g}^{2}=4 \pi$. The small brown color region shows present reach of the mass scales based on combined results from $6 \mathrm{GeV}$ PVDIS and other precision experiments [11] while the large orange color region is the expanded sensitivity assuming final precision from Qweak [2] and SoLID PVDIS.

kinematic range. The proposed two different electron beam energies, at $11 \mathrm{GeV}$ and $6.6 \mathrm{GeV}$, will provide range of $\mathrm{Q}^{2}$ values for each $\mathrm{x}_{\mathrm{bjk}}$.

The cryogenic solenoidal magnet from CLEO-II experiment will be refurbished and implement as the SoLID magnet after certain modifications to match SoLID specifications. Gas Electron Multiplier detectors (GEMs) will be used as tracking detectors implemented within the solenoidal magnetic field and in downstream of the magnet where main particle detectors are located [12]. The light gas Cerenkov and the electromagnetic calorimeter will primarily be used to particle identification and reject the pion background [12]. The proposed data acquisition (DAQ) system will be based on pipe-lined electronics for triggering and data readout to accommodate very high rates of above $1 \mathrm{MHz}$. The detectors will be triggered and readout independently for azimuthally separated sectors to increase the readout rate. An upgraded Compton polarimeter and a superconducting Møller polarimeter will both assumed to be readily available by the time SoLID program will start taking data.

\section{Summary}

The SoLID apparatus is design to have a broad physics program. The PVDIS program discussed in the proceeding is only a part of this physics program. The semi-inclusive deep inelastic scattering (SIDIS) program using SoLID will measure single and double spin asymmetries to ac- 


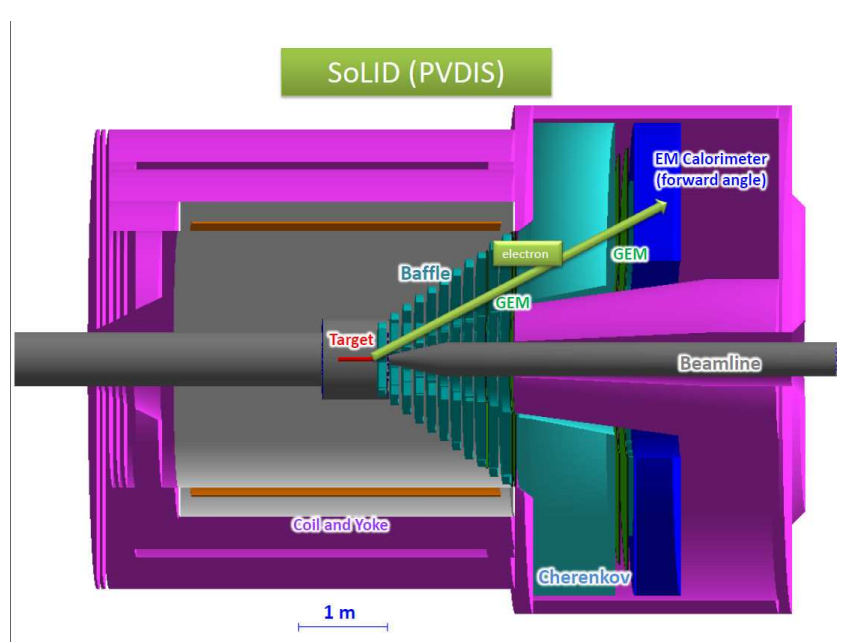

Figure 5: The SoLID apparatus for PVDIS configuration with the target, baffle/collimation system, coil and york of the solenoidal magnet, and the system detectors is shown.
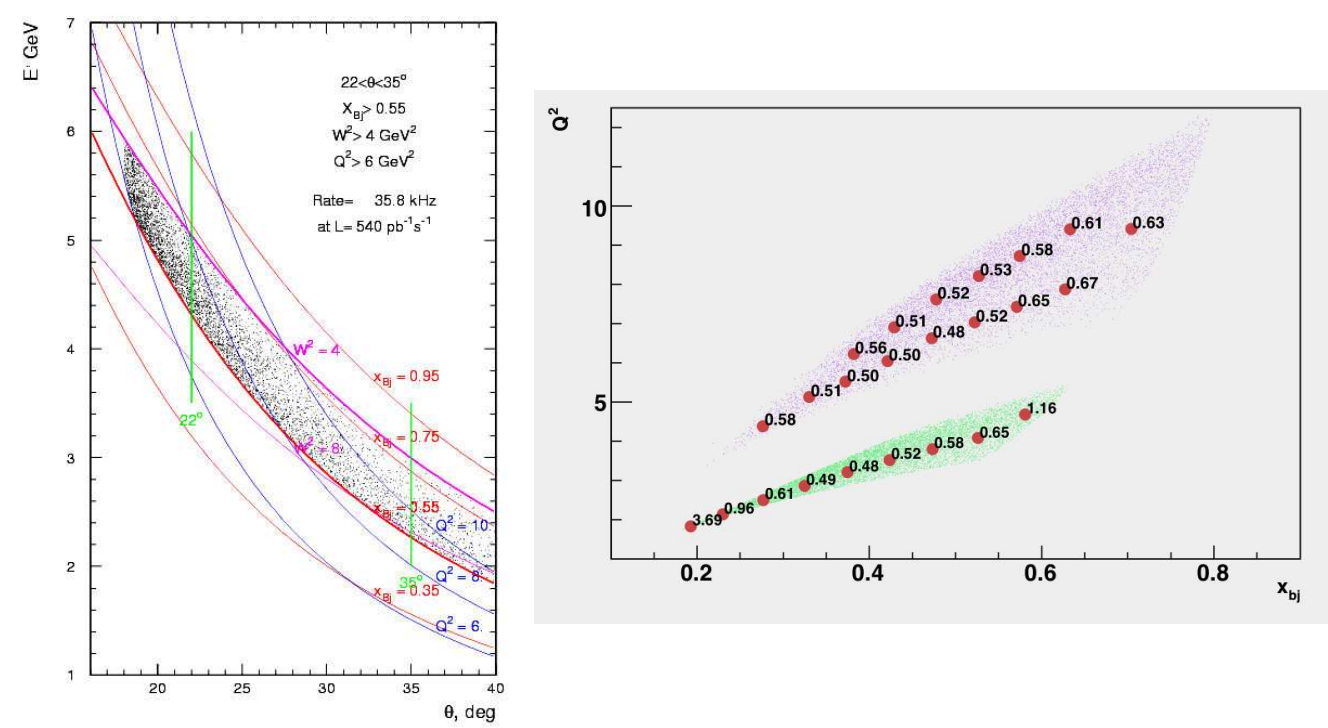

Figure 6: The kinematic reach of the SoLID apparatus for $11 \mathrm{GeV}$ electron beam is shown in electron momentum vs. scattering angle in the left plot. In the plot at right, the statistical uncertainty of the PVDIS asymmetry in $\mathrm{Q}^{2}$ and $\mathrm{x}_{\mathrm{bjk}}$ bins are shown for 4 months at $11 \mathrm{GeV}$ (violet band) while 2 months at $6.6 \mathrm{GeV}$ (green band), respectively.

cess transverse momentum dependent parton distributions (TMD) and allow reconstruction of the nucleon structure in three dimension momentum space [13]. The $\mathrm{J} / \psi$ program will measure differential electroproduction cross sections of $\mathrm{J} / \psi$ near threshold on a proton as a function of the four-momentum transfer to the nucleon. The $\mathrm{J} / \psi$ program will help to resolve the conformal anomaly, origin of the large fraction of nucleon mass and test different model interpretations of the anomaly [14].

The SoLID apparatus was first proposed in 2009. Since then there have been many develop- 
ment in conceptual design, Monte-Carlo simulation, detector R\&D and acquiring infrastructure. We have performed initial engineering feasibility studies on the compatibility and refurbishment required for the CLEO-II magnet to be used as the solenoidal magnet. The Jefferson Lab management is currently in the process of transporting the CLEO-II magnet. Recently SoLID collaboration successfully completed most up-to date pre-conceptual design review [15] by physics division of the Jefferson Lab.

\section{References}

[1] K.S. Kumar, Sonny Mantry, W.J. Marciano, and P.A. Souder. Low Energy Measurements of the Weak Mixing Angle. Ann.Rev.Nucl.Part.Sci., 63:237-267, 2013.

[2] D. Androic et al. First determination of the weak charge of the proton. Phys. Rev. Lett., 111:141803, Oct 2013.

[3] J. Beringer et al. Review of particle physics. Phys. Rev. D, 86:010001, Jul 2012.

[4] P.L. Anthony et al. Precision measurement of the weak mixing angle in Moller scattering. Phys.Rev.Lett., 95:081601, 2005.

[5] G.P. Zeller et al. A Precise Determination of Electroweak Parameters in Neutrino Nucleon Scattering. Phys.Rev.Lett., 88:091802, 2002.

[6] Juliette Mammei. The MOLLER Experiment. Nuovo Cim., C035N04:203-208, 2012.

[7] I. C. Cloët, W. Bentz, and A. W. Thomas. Parity-violating deep inelastic scattering and the flavor dependence of the emc effect. Phys. Rev. Lett., 109:182301, Oct 2012.

[8] J. Arrington, J. G. Rubin, and W. Melnitchouk. How well do we know the neutron structure function? Phys. Rev. Lett., 108:252001, Jun 2012.

[9] N. Baillie et al. Measurement of the neutron $F_{2}$ structure function via spectator tagging with clas. Phys. Rev. Lett., 108:142001, Apr 2012.

[10] Sonny Mantry, Michael J. Ramsey-Musolf, and Gian Franco Sacco. Examination of higher-order twist contributions in parity-violating deep-inelastic electron-deuteron scattering. Phys. Rev. C, 82:065205, Dec 2010.

[11] D. Wang et al. Measurement of parity violation in electronâĂŞquark scattering. Nature, 506(7486):67-70, 2014.

[12] The SoLID Collaboration. Precision Measurement of Patiry-violation in Deep Inelastic Scattering Over a Broad Kinematic Range. Technical report, Proposal to Jefferson Lab PAC35: https://www.jlab.org/exp_prog/proposals/10/PR12-10-007.pdf, December 2009.

[13] The SoLID Collaboration. Target Single Spin Asymmetry in Semi-Inclusive Deep-Inelastic (e, $\left.e^{\prime} \pi^{ \pm}\right)$ Reaction on a Transversely Polarized Proton Target. Technical report, Proposal to Jefferson Lab PAC38: http://www.jlab.org/exp_prog/proposals/11/PR12-11-108.pdf, July 2011.

[14] The SoLID Collaboration. Near Threshold Electroproduction of J/Psi at $11 \mathrm{GeV}$. Technical report, Proposal to Jefferson Lab PAC39: http://www.jlab.org/exp_prog/proposals/12/PR12-12-006.pdf, July 2012.

[15] The SoLID Collaboration. Solid preliminary conceptual design report. Technical report, Jefferson Lab : http://hallaweb.jlab.org/12GeV/SoLID/dowload/doc/solid_precdr.pdf, May 2014. 